Pathologe 2021 - 42 (Suppl 2):S214-S215 https://doi.org/10.1007/s00292-021-00990-1 Angenommen: 3. September 2021 Online publiziert: 4. Oktober 2021 (c) Springer Medizin Verlag $\mathrm{GmbH}$, ein Teil von Springer Nature 2021

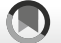

\section{Erwin Georg Dahme}

30.06.1926-05.05.2021

Walter Hermanns · Kaspar Matiasek · Rüdiger Wanke

Institut für Tierpathologie, Ludwig-Maximilians-Universität München, München, Deutschland

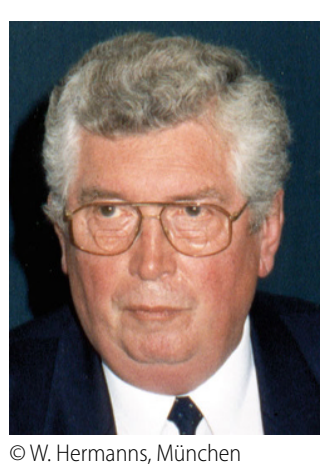

Am 5. Mai 2021 verstarb Herr Professor Dr. Dr. honoris causa Erwin Georg Dahme in seinem 95. Lebensjahr in Germering bei München.

Erwin Dahme wurde am 30. Juni 1926 in Bamberg geboren. Er besuchte die Volksschule in München (1932-36), die Katholische Oberschule am Holzdamm und die Jungen-Oberrealschule Eppendorf in Hamburg (1936-41) sowie ab 1941 das Giesela-Gymnasium in München, das er 1944 mit einem Notabitur abschloss. Bereits ab 1943 war E. Dahme als Luftwaffenhelfer verpflichtet, es folgten der Reichsarbeitsdienst und die Wehrmachtszeit bei den Gebirgsjägern bis zu seiner Entlassung durch die US-Armee im Juli 1945.

Ab dem Wintersemester 1945/46 nahm Erwin Dahme das Studium der Tiermedizin in München auf, das er im Sommersemester 1950 mit dem Tierärztlichen Examen beendete. Von Oktober 1950 bis Ende 1953 war er als wissenschaftlicher Assistent im Institut für Tierpathologie tätig. In dieser Zeit (1951) promovierte er zum Dr. med. vet. bei Professor Hans Sedlmeier mit der Arbeit „Morphologische Veränderungen bei den Nephritiden des Huhnes". Ab 1954 war E. Dahme für einige Monate am Pharmakologisch-Gewerbehygienischen Institut der BASF in Ludwigs- hafen tätig und danach für knapp ein Jahr als Consultant der Veterinary Public Health Division der World Health Organization (WHO) in Genf als Nachfolger von Hugo Stünzi, Zürich. Im Jahr 1955 nahm er seine wissenschaftliche Tätigkeit am Institut für Tierpathologie der LMU wieder auf und habilitierte sich 1956 mit der Schrift "Über die Beurteilung der Angiopathien bei chronisch-sklerosierenden Nierenkrankheiten des Hundes" für das Fachgebiet „Allgemeine Pathologie, pathologische Anatomie und Histologie".

Nach der Ernennung zum Privatdozenten (1957) folgten die Ernennungen zum außerplanmäßigen Professor (März 1963) und schließlich die Berufung zum ordentlichen Professor am 1. März 1965. Gleichzeitig wurde er mit der Leitung des 1964 neu gegründeten Instituts für Onkologie und Neuropathologie betraut, welches ab 1969 unter dem Namen Institut für Allgemeine Pathologie und Neuropathologie geführt wurde. Den gleichnamigen Lehrstuhl hatte Erwin Dahme dann nahezu 30 Jahre bis zu seiner Emeritierung am 30. September 1994 inne. Die Zusammenlegung mit dem zweiten Lehrstuhl zum Institut für Tierpathologie erfolgte nach dem Dezember 1973. Professor Dahme ist damit Begründer der ersten spezialisierten veterinärneuropathologischen Einrichtung im deutschsprachigen Raum und wurde so zu einem der Nestoren dieser Disziplin in Europa.

Erwin Dahme war stets bemüht, in enger Zusammenarbeit mit humanmedizinischen Kollegen, die speziesübergreifenden Aspekte spontaner wie experimenteller Krankheiten bei Tieren bezüglich ihres Modellcharakters für Krankheiten des Menschen zu hinterfragen. 
Das Spektrum seines Schaffens spannt sich von Studien zur diaplazentaren Toxoplasma-Infektion im Jahre 1952 bis zur Erstbeschreibung einer Nemalinkörpermyopathie beim Kalb im Jahre 1997. Es waren insbesondere 3 Themenschwerpunkte, die sein wissenschaftliches Arbeiten bestimmten: Neuropathologie, Gefäßpathologie und die Onkologie. Dabei sah er das Nervensystem nicht als ein isoliertes Organsystem an, sondern er "hangelte sich" - eigenen Worten nach über sein Interesse an der Gefäßpathologie „in das Hirn hinein“. Entscheidende Anregungen empfing er dabei von seinen Mentoren Professor Dr. Hans Sedlmeier und dem ehemaligen Direktor des Nervenkrankenhauses Haar, Herrn Professor Dr. Anton von Braunmühl. Der Einblick in die Laborarbeiten von Braunmühls waren wegweisend für den jungen Forscher Dahme und weckte sowohl sein Interesse an neurovaskulären Krankheiten wie auch an der vergleichenden Alterspathologie. Bei Letzterer waren es besonders die Proteinfehlfaltungskrankheiten, die Amyloidosen und Prionenerkrankungen wie die spon$\tan$ bei Schafen auftretende Scrapie. So war er ein kompetenter Ansprechpartner, als im Jahre 1989 die ersten Fälle von boviner spongiformer Enzephalopathie in Deutschland auftraten. Hier war Professor Dahme nicht nur als Diagnostiker, sondern vor allem auch als gesundheitspolitischer Sachverständiger gefragt, der sich gleichermaßen in der Verantwortung für Mensch und Haustier sah.

Professor Dahme hat an rund 200 Publikationen mitgewirkt, mehr als 70 Dissertationen betreut sowie insgesamt 5 Kolleginnen und Kollegen habilitiert, von denen 3 auf Lehrstühle berufen wurden (in Hamburg, München und Wien). Er war Mitautor oder Herausgeber von Handbüchern der speziellen Veterinärpathologie und von Lehrbüchern in allgemeiner Pathologie, in spezieller Pathologie und in pathologischer Histologie, vielfach in mehreren Auflagen.

Im September 1994 wurde Professor Dahme aufgrund seiner vielfältigen fachlichen Verdienste die Ehrendoktorwürde der Tierärztlichen Hochschule Hannover „in Anerkennung seiner vorbildlichen wissenschaftlichen Leistungen auf den Gebieten der Gefäßpathologie, der Onkologie sowie der vergleichenden Neuropathologie, zugleich in Würdigung seines erfolgreichen Wirkens als Hochschullehrer und Verfasser von Lehrbüchern der Allgemeinen und Speziellen Veterinärpathologie sowie seines unermüdlichen Einsatzes für die tierärztliche Fortbildung und Förderung des wissenschaftlichen Nachwuchses" verliehen.

Neben seinen Aufgaben als Ordinarius hat sich Herr Professor Dahme uneingeschränkt der akademischen Selbstverwaltung zur Verfügung gestellt. So wirkte er 1972-74 als Dekan der Tierärztlichen Fakultät und er war 5 Jahre als Vertreter der Fakultät im Akademischen Senat und mehrere Jahre in der Versammlung der LMU tätig. Für die Deutsche Forschungsgemeinschaft war Herr Dahme viele Jahre als Fachgutachter tätig.

Mitglied der Deutschen Gesellschaft für Pathologie wurde Professor Dahme bereits im Jahre 1953, zu einer Zeit, als die deutschsprachigen Veterinärpathologen gerade begonnen hatten, sich in der Arbeitsgemeinschaft der Veterinärpathologen zu organisieren. In den Jahren 1989 und 1990 war Herr Dahme Präsident der Deutschen Gesellschaft für Neuropathologie und Neuroanatomie und im Oktober 2013 ernannte ihn das European College of Veterinary Neurology aufgrund seiner Lebensleistungen zu seinem Ehrenmitglied.

Besondere Verdienste hat sich Prof. Dahme auch als Vertreter der Tierärztlichen Fakultät der LMU in der Bayerischen Landestierärztekammer (BLTK) erworben. Er war langjähriges Mitglied des Weiter- und des Fortbildungsausschusses. Von 1995 bis 1999 war er Delegierter und von 1970 bis 1991 Vorstandsmitglied in der BLTK. Sein herausragendes Engagement hat die Bayerische Landestierärztekammer durch die Verleihung des Goldenen Ehrenzeichens und mit der Ehrenmitgliedschaft gewürdigt. In den Jahren 1994-2004 diente er außerdem dem Landesgesundheitsrat des Freistaates Bayern als Vertreter des Bereichs Tiermedizin. Erwähnt sei auch die Leitung der bereits 1873 gegründeten Münchner Tierärztlichen Gesellschaft, heute würde man von Alumnivereinigung sprechen, deren Vorsitzender er von 1978 bis 1991 war.
Mit Herrn Professor Dr. Dr. h. c. Erwin Dahme verliert die Pathologie eines ihrer fachkompetentesten Mitglieder, das sich als eloquenter Polyhistor persönlich durch seine Großzügigkeit, Gelassenheit, Bescheidenheit und seinen unverbesserlichen Humor ausgezeichnet hat. Alle die ihn kannten, werden ihm stets ein ehrendes Andenken bewahren.

The supplement containing this article is not sponsored by industry.

\section{Korrespondenzadresse}

Prof. Dr. Walter Hermanns

Institut für Tierpathologie, Ludwig-

Maximilians-Universität München

Veterinärstraße 13, 80539 München,

Deutschland

hermanns@patho.vetmed.uni-muenchen.de 